\title{
FLORA POLINICA DE CHAMELA, JALISCO (FAMILIAS AMARANTHACEAE, COMBRETACEAE, LOASACEAE, MARTYNIACEAE, PAPAVERACEAE, TILIACEAE Y VIOLACEAE) ${ }^{1}$
}

\author{
DaVID LEONOR QUIROZ-GARCIA \\ Rodolfo Palacios-Chavez ${ }^{2}$ \\ Ma. de la Luz Arreguin-Sanchez ${ }^{2}$ \\ Departamento de Botánica \\ Escuela Nacional de Ciencias Biológicas \\ Instituto Politécnico Nacional \\ Plan de Ayala y Carpio, Col. Santo Tomás \\ 11340 México, D.F.
}

\begin{abstract}
RESUMEN
Se estudia e ilustra la morfología de los granos de polen de 7 familias y 21 especies de la Estación de Biología Chamela, Jalisco, de la Universidad Nacional Autónoma de México, a mencionar: Amaranthaceae que incluye a Achyranthes aspera L., Alternanthera cf. pycnantha (Benth.) Standl., Amaranthus retroflexus L., Chamissoa altissima (Jacq.) H.B.K., Froelichia interrupta (L.) Moq., Gomphrena decumbens Jacq., Iresine calea (Ibáñez) Standl., I. interrupta Benth. y Lagrezia monosperma (Rose) Standl.; Combretaceae, representada por Combretum fruticosum (Loefl.) Stuntz y C. mexicanum Humb. et Bonpl.; Loasaceae por Gronovia scandens L. y Mentzelia aspera L.; Loganiaceae por Strychnos cf. brachistantha Standl.; Martyniaceae por Martynia annua L.; Papaveraceae por Argemone ochroleuca Sweet subsp. ochroleuca; Tiliaceae por Heliocarpus pallidus Rose, Luehea candida (DC.) Mart. y Triumfetta acracantha Hochr., Violaceae por Hybanthus attenuatus (Humb. \& Bonpl.) G. K. Schulze, H. mexicanus Ging. y H. serrulatus Standl.

Las observaciones de los granos de polen se realizaron con el microscopio de luz y microscopio electrónico de barrido. En las familias Amaranthaceae, Loasaceae y Tiliaceae es posible diferenciar por medios palinológicos algunos de los taxa estudiados.
\end{abstract}

\section{ABSTRACT}

Pollen grains morphology of 7 families and 21 species from the Estación de Biología Chamela, Jalisco, of the Universidad Nacional Autónoma de México is studied and illustrated. The following taxa are included: Amaranthaceae represented by Achyranthes aspera L., Alternanthera cf. pycnantha (Benth.) Standl., Amaranthus retroflexus L., Chamissoa altissima (Jacq.) H.B.K., Froelichia interrupta (L.) Moq., Gomphrena decumbens Jacq., Iresine calea (Ibáñez) Standl., I. interrupta Benth. and Lagrezia monosperma (Rose) Standl.; Combretaceae by Combretum fruticosum (Loefl.) Stuntz and C. mexicanum (L.) Mow.; Loasaceae by Gronovia scandens L. and Mentzelia aspera L.; Loganiaceae by Strychnos cf. brachistantha Standl.; Martyniaceae by Martynia annua L.; Papaveraceae by Argemone ochroleuca

\footnotetext{
${ }^{1}$ Trabajo parcialmente subsidiado por la Dirección de Estudios de Posgrado e Investigación del Instituto Politécnico Nacional (870285) y por el Smithsonian Tropical Research Institute.

${ }^{2}$ Becarios de COFAA del I.P.N.
} 
subsp. ochroleuca Sweet; Tiliaceae by Heliocarpus pallidus Rose, Luehea candida (DC.) Mart. and Triumfetta acracantha Hochr.; Violaceae by Hybanthus attenuantus (Humb. \& Bonpl.) G. K. Schulze, $H$. mexicanus Ging. and $H$. serrulatus Standl.

The observations of pollen grains were carried out with scanning electron microscopy and light microscopy. Taxonomic keys for separation of genera and species of pollen grains in the Amaranthaceae, Loasaceae and Tiliaceae are included.

\section{INTRODUCCION}

El presente trabajo forma parte de los estudios sobre la flora polínica de Chamela, Jalisco, que Palacios-Chávez et al. (1986) vienen realizando. Su objetivo es dar a conocer la morfología del polen de las diferentes plantas de la región, lo que será de utilidad en investigaciones sobre paleobotánica, lluvias de polen actual y fósil y en la interpretación taxonómica y evolutiva de los diferentes géneros y especies. También servirán de ayuda a los trabajos de ecología de la polinización que se desarrollan en este lugar.

\section{METODOLOGIA}

El polen en su mayor parte fue desprendido de los ejemplares de herbario depositados en el museo de la Estación de Biología Chamela, Jalisco. Cuando no se pudo tomar polen de esa colección por carecer de él, el material se obtuvo de ejemplares de otras partes del país previa corroboración de la identificación de los mismos. Las muestras polínicas se dividieron en dos partes; una fue tratada con la técnica de acetólisis de Erdtman (1943) para observaciones al microscopio de luz (ML) y otra parte, sin tratamiento químico, se utilizó para el microscopio electrónico de barrido (MEB). El polen fue sombreado con Au para observaciones en un microscopio JEOL-JSM 35.

\section{RESULTADOS}

Descripción de los granos de polen

Familia Amaranthaceae

Taxonomía: Lott (1985), Schinz (1934).

Palinología: Erdtman (1966), Huang (1972), Markgraf \& D’Antoni (1978), Moncada (1987), Nowicke \& Skvarla (1979), Riollet \& Bonnefille (1976), Tsukada (1967).

Achyranthes aspera L.

Estación de Biología Chamela, Jalisco. S. H. Bullock 1571 (MEXU). Lám. I. Figs. 1 a 3.

Polen periporado, tectado, esférico de 16.9 (18.5) $19.5 \mu$ de diámetro. Exina de 1.4 $\mu$ de grosor, con la sexina de mayor espesor que la nexina, superficialmente al ML y al MEB se aprecia equinulada. Poros \pm 40 , circulares de $2.5 \mu$ de diámetro con membranas microequinadas. 
Alternanthera cf. pycnantha (Benth.) Standl. Estación de Biología Chamela, Jalisco.

E. Lott 2771 (MEXU).

Lám. I. Figs. 4 a 6.

Polen periporado, semitectado, esferoidal-poliédrico de 18.2 (21.2) 21.2 X 17.8 (21) $22.5 \mu$. Exina de $1.6 \mu$ de grosor, con la sexina de mayor espesor que la nexina, superficialmente al ML se aprecia reticulada-fenestrada, doce fenestras pentagonales de 7.8 (8.7) $9.1 \mu$ de diámetro y muros de $2 \mu$ de ancho, con pequeñas espinas supratectales. Poros 12, circulares de $7.6 \mu$ de diámetro dentro de las fenestras.

Amaranthus retroflexus L.

Municipio La Huerta, Chamela, Jalisco.

Estación de Biología Chamela.

E. Lott 1215 (MEXU).

Lám. I. Figs. 7 a 10.

Polen periporado, tectado, esferoidal de 26 (27.6) 28.6 X 28.6 (29.3) $29.9 \mu$. Exina de $1.3 \mu$ de grosor, con la sexina de igual espesor que la nexina, superficialmente al ML y al MEB se aprecia equinulada. Poros \pm 50 , circulares de $2.6 \mu$ de diámetro, cubiertos con opérculos microequinados.

Chamissoa altissima (Jacq.) H.B.K. L La Concepción, Mpio. Jilotepec, Veracruz. F. Ventura 10864 (ENCB).

Lám. II. Figs. 1 a 4.

Polen periporado, tectado, esferoidal de 26 (28) $29.9 \times 26$ (27.7) $29.9 \mu$. Exina de $2 \mu$ de grosor, con la sexina de mayor espesor que la nexina, superficialmente al ML y MEB se aprecia equinulada. Poros \pm 24 , circulares de $3.5 \mu$ de diámetro, cubiertos con opérculos microequinados.

Froelichia interrupta (L.) Moq.

Cerca de la costa, entre San Lucas

y San José del Cabo, Baja California Sur. J. H. Thomas 8496 (ENCB).

Lám. II. Figs. 5 a 8.

Polen periporado, semitectado, esferoidal de 33.8 (39.8) 45.5 X 32.5 (39.9) $44.2 \mu$. Exina de $5.2 \mu$ de grosor, con la sexina de mayor espesor que la nexina, superficialmente al ML y MEB se aprecia per-reticulada con pequeñas espinas sobre los muros, las lúminas son poligonales de 2.6 (3.9) $5.2 \times 3.9 \mu$. Poros \pm 100 , circulares de $1.9 \mu$ de diámetro situados en el centro de las lúminas, cubiertos con opérculos microequinados.

Gomphrena decumbens Jacq.

Alrededores de La Salitrera, Mpio. de Zaragoza, San Luis Potosí. J. Rzedowski 11426 (ENCB).

Lám. II. Figs. 9 a 12.

Polen periporado, semitectado, esferoidal de 18.2 (22.2) $26.0 \times 19.5$ (22) $24.7 \mu$. Exina de $3.4 \mu$ de grosor, con la sexina de mayor espesor que la nexina, superficialmente 
al ML y MEB se aprecia per-reticulada, las lúminas son poligonales de 2.6 (3.6) 3.9 X 2.6 $\mu$ y los muros de $\pm 1 \mu$ de grosor. Poros \pm 60 , circulares de $2.2 \mu$ de diámetro, situados en el centro de las lúminas.

Iresine calea (Ibáñez) Standl.

$6 \mathrm{~km}$ al NW de Jonacatepec, Morelos.

L. González 3636 (ENCB).

Lám. III. Figs. 1 a 4.

Polen periporado, tectado-perforado, esférico de 15.6 (17.9) $19.5 \mu$ de diámetro. Exina de $2.2 \mu$ de grosor, con la sexina de mayor espesor que la nexina, superficialmente al ML y MEB se aprecia equinulada. Poros \pm 23 , circulares de $2.8 \mu$ de diámetro, cubiertos con opérculos microequinados.

Iresine interrupta Benth.

Camino de Chiapa de Corzo a

Pichucalco, Chiapas.

W. R. Anderson 5531 (ENCB).

Lám. III. Figs. 5 a 8.

Polen periporado, tectado-perforado, esférico-poligonal de 13 (14.6) $19.5 \mu$ de diámetro. Exina de $1.3 \mu$ de grosor, con la sexina de mayor espesor que la nexina, superficialmente al ML se aprecia equinulada y al MEB equinulada con perforaciones. Poros \pm 25 , circulares de $2.2 \mu$ de diámetro, cubiertos con opérculos microequinados.

Lagrezia monosperma

(Rose) Standl.
$1 \mathrm{~km}$ al SW de Tomatlán, Jalisco.

J. Rzedowski 17778 (ENCB).

Lám. III. Figs. 9 a 12.

Polen periporado, tectado-perforado, esférico-poligonal de 18.2 (20) $20.8 \mu$ de diámetro. Exina de $1.5 \mu$ de grosor, con la sexina de mayor espesor que la nexina, superficialmente al ML se aprecia equinulada y al MEB equinulada con perforaciones. Poros \pm 20 , circulares de $3.3 \mu$ de diámetro, cubiertos con opérculos microequinados.

Clave para diferenciar los taxa de la familia Amaranthaceae

1. Polen tectado

2. Polen con poros numerosos, cerca de 50 Achyranthes aspera Lám. I. Figs. 1 a 3 Amaranthus retroflexus Lám. I. Figs. 7 a 10

2. Polen con no más de 30 poros Chamissoa altissima Lám. II. Figs. 1 a 4 Iresine calea Lám. III. Figs. 1 a 4 I. interrupta Lám. III. Figs. 5 a 8 Lagrezia monosperma Lám. III. Figs. 9 a 12 
1. Polen semitectado

3. Polen reticulado-fenestrado, con 12 poros Alternanthera cf. pycnantha Lám. I. Figs. 4 a 6

3. Polen per-reticulado, con numerosos poros

4. Poros \pm 60 Gomphrena decumbens Lám. II. Figs. 9 a 12

4. Poros más de 100 Froelichia interrupta Lám. II. Figs. 5 a 8

Comentarios

El polen de la familia Amaranthaceae se separa en dos grupos: a) el tectado, periporado, equinulado presente en Achyranthes aspera, Amaranthus retroflexus, Chamissoa altissima, Iresine calea, I. interrupta y Lagrezia monosperma y b) semitectado, periporado, reticulado o fenestrado encontrado en Alternanthera cf. pycnantha, Froelichia interrupta y Gomphrena decumbens. De estos grupos el primero es el que muestra semejanza con las microsporas de la familia Chenopodiaceae.

Al relacionar la morfología del polen con la clasificación taxonómica de las Amaranthaceae propuesta por Schinz (1934), se encontró lo siguiente: este autor reconoce dos subfamilias, las Amaranthoideae, en donde sitúa a los géneros Achyranthes, Amaranthus, Chamissoa y Lagrezia y las Gomphrenoideae, con Alternanthera, Froelichia, Gomphrena e Iresine. Sin embargo, esta separación no es apoyada por los tipos polínicos determinados, en los cuales Iresine por las características de su polen se reune con los géneros de la subfamilia Amaranthoideae y no con los de la Gomphrenoideae.

Las descripciones del polen de amarantáceas, realizadas por otros autores, concuerdan en general con las de esta investigación, excepto en el caso de Iresine, pues Nowicke \& Skvarla (1979) y Moncada (1987) lo describen como semejante al tipo Gomphrena. Estas discrepancias parecen tener su explicación en lo que apunta Erdtman (1966) en el sentido de que el polen de Iresine aunque se incluye en el tipo Amaranthus, muestra características que lo señalan como una transición hacia el tipo Gomphrena.

Familia Combretaceae

Taxonomía: Lott (1985).

Palinología: Association des Palinologues de Langue Française (1974), Barth \& Silva (1965), Erdtman (1966), Graham (1980), Kubitzki (1965), Maley (1970), Melhem \& Paula (1966).

Combretum fruticosum (Loefl.) Stuntz

Estación de Biología Chamela, Jalisco.

S. H. Bullock 1324 (MEXU).

Lám. IV. Figs. 1 a 7.

Polen heterocolpado, tricolporado, tectado, esferoidal de 24 (26) $28 \times 23$ (24) 25 $\mu$. Vista polar interhexagonal de 20 (23) $25 \mu$ de diámetro. $P / E=1.0$. Exina de $2 \mu$ de grosor, con la nexina ligeramente más gruesa que la sexina, superficialmente al MEB se observa levemente estriada con microfoveolos o foveolos menores de $1 \mu$ y al $M L$ se aprecia escabrosa. Colpos verdaderos de 20 (23) $25 \mu$ de largo por $\pm 2 \mu$ de ancho, con las membranas lisas y una fisura más delgada, a veces difusos. Poros circulares de $3.5 \mu$ de diámetro. Indice del área polar 0.25 , pequeña. 
Combretum mexicanum Humb. et Bonpl. Estación de Biología Chamela, Jalisco. S. H. Bullock 1580 (MEXU). Lám. IV. Figs. 8 a 14.

Polen heterocolpado, tricolporado, tectado, esferoidal, de 17 (21.7) $23 \times 17$ (19) 20 $\mu$. Vista polar interhexagonal de 16 (19) $21 \mu$ de diámetro. $P / E=1.1$. Exina de $2 \mu$ de grosor con la sexina de igual espesor que la nexina; superficialmente al MEB se aprecia estriada y al ML las estrias son difusas. Colpos verdaderos de 16 (17.5) $19 \mu$ de largo por $2 \mu$ de ancho, con las membranas lisas alternando con tres pseudocolpos los cuales son mucho más cortos y más delgados que los colpos verdaderos. Poro circular de 3 a $4 \mu$ de diámetro. Indice del área polar 0.10 , pequeña.

\section{Comentarios}

Las descripciones de los granos de polen de estas especies coinciden con las de los demás autores, a excepción de Graham (1980) quien discute que por la semejanza que tienen con los de Terminalia son difíciles de diferenciar al microscopio de luz, y sólo pueden ser separados cuando se observan al microscopio electrónico de barrido. En el caso de Combretum fruticosum y $C$. mexicanum se repite esta situación, ya que unicamente a mayores aumentos se observan diferencias en la ornamentación.

Familia Loasaceae

Taxonomía: Lott (1985).

Palinología: Erdtman (1966), Heusser (1971).

Gronovia scandens L.

Estación de Biología Chamela, Jalisco. E. Lott 610 (MEXU).

Lám. V. Figs. 1 a 6.

Polen tricolporado, tectado, esférico, de 24 (27.5) $32 \times 24$ (27) $30.4 \mu$. Vista polar circular de 24 (27.6) $30.4 \mu$ de diámetro. $P / E=1.0$. Exina de $2 \mu$ de grosor, sexina y nexina de igual espesor, superficialmente al MEB y al ML se aprecia equinulada. Colpos de 20 (22.4) $27 \mu$ de largo por 1.5 a $3 \mu$ de ancho, con los márgenes dentados y membranas equinuladas. Indice del área polar 0.22 , pequeña.

Mentzelia aspera L.

Estación de Biología Chamela, Jalisco.

S. H. Bullock 1262 (MEXU).

Lám. V. Figs. 7 a 12.

Polen tricolporado, semitectado, prolato de 35 (42) $44 \times 23$ (26) $28 \mu$. Vista polar circular de 22 (25) $28 \mu$ de diámetro. $P / E=1.6$. Exina de $2.5 \mu$ de grosor, engrosándose en los polos hasta 4 micras, superficialmente se aprecia estriada-reticulada al ML y MEB. Colpos de 24 (30.5) $39 \mu$ de largo por 3 a $5 \mu$ de ancho, con membranas equinuladas. Poros de 6 a $8 \mu$ por 3 a $5 \mu$, difusos con costa colpi. Indice del área polar 0.24, pequeña. 
Clave para diferenciar las especies de la familia Loasaceae

1. Polen tricolpado, equinulado, esférico Gronovia scandens Lám. V. Figs. 1 a 6

1. Polen tricolporado, estriado-reticular, prolato Mentzelia aspera

Lám. V. Figs. 7 a 12

\section{Comentarios}

Las descripciones de los granos de polen pertenecientes a esta familia no coinciden con la de Erdtman (1966), quien registra para Gronovia longiflora una ornamentación con proyecciones romas, ni tampoco con la de Heusser (1971), pues el polen de Mentzelia chilensis, aunque coincide con las demás características aquí dadas, su ornamentación es considerada como finamente reticulada.

Familia Loganiaceae

Taxonomía: Lott (1985).

Palinología: Erdtman (1966), Punt \& Leenhouts (1967), Valio \& Labouriau (1964).

Strychnos cf. brachistantha Standl. Estación de Biología Chamela, Jalisco.

A. Pérez 1943 (MEXU).

Lam. VI. Figs. 1 a 5.

Polen tricolporado, tectado, esférico, de 21 (25.8) $30 \times 20$ (25.5) $30 \mu$. Vista polar semi-angular de 20 (24) $26 \mu$ de diámetro. $P / E=1.0$. Exina de $1.6 \mu$ de grosor, con la sexina de igual espesor que la nexina, superficialmente al MEB se observa punteada con escabras aisladas, al ML levemente escabrosa. Colpos de 16 (18) $23 \mu$ de largo por $1.6 \mu$ de ancho, con membranas lisas. Indice del área polar 0.30, mediana.

Comentarios

Erdtman (1966) menciona que algunas especies de Strychnos muestran granos de polen finamente reticulados, lo que no se pudo observar en las microsporas aquí estudiadas.

Familia Martyniaceae

Taxonomía: Lott (1985).

Palinología: Erdtman (1966), Ludlow-Wiechers \& Roldán-Ramos (1984).

Martynia annua L. Estación de Biología Chamela, Jalisco.

J. A. Solís 768 (MEXU).

Lám. VI. Figs. 6 a 11.

Polen pericolpado, semitectado, esférico de 81 (84.5) $100 \mu$ de diámetro. Exina de $4 \mu$ de grosor, con la nexina ligeramente más gruesa que la sexina; al MEB se observan procesos equinulados sobre los muros y al ML se aprecia per-reticular. Colpos dispuestos 
en forma de pentágonos sobre la superficie del grano con medidas entre 20 y $25 \mu$ de largo por $\pm 2.5 \mu$ de ancho.

Comentarios

La morfología del polen de este taxon coincide con la señalada en las descripciones de los autores arriba mencionados.

Familia Papaveraceae

Taxonomía: Ownbey (1958), Lott (1985).

Palinología: Erdtman (1966), Jain \& Nanda (1966), Nair \& Sharma (1962).

Argemone ochroleuca Sweet

subsp. ochroleuca
Estación de Biología Chamela, Jalisco. S. H. Bullock 1877 (MEXU).

Lám. VII, Figs. 1 a 8.

Polen tricolpado, algunas veces heteropolar, sincolpado, semitectado, esferoidal de 34 (36.7) 39 X 35 (37) $39 \mu$. Vista polar circular de 35 (37) $39 \mu$ de diámetro. $P / E=0.9$. Exina de $2.5 \mu$ de grosor, con la nexina mucho más delgada que la sexina; superficialmente al MEB se observan procesos equinulados sobre los muros y al ML se aprecia reticulada. Colpos de 28 (29.6) $34 \mu$ de largo por 4 a $8 \mu$ de ancho, con las membranas escabrosas, algunos coalescentes en uno de los polos. Indice del área polar 0.16 , pequeña.

\section{Comentarios}

El polen de Argemone ochroleuca subsp. ochroleuca aquí descrito presenta ligeras diferencias con el de otras especies de este género, principalmente en lo que a ornamentación se refiere, pues varios autores lo consideran como levemente reticulado, en cambio en el de Chamela, los retículos son claros y bien definidos.

Familia Tiliaceae

Taxonomía: Lott (1985).

Palinología: Erdtman (1966), Huang (1972), Kapp (1969), Palacios (1966), Plá Dalmau (1961).

Heliocarpus pallidus Rose

Estación de Biología Chamela, Jalisco. S. H. Bullock 1480 (MEXU).

Lám. VII. Figs. 9 a 12.

Polen tricolporado, tectado, prolato de 49 (51) $55 \times 27$ (28) $31.5 \mu$. Vista polar poco frecuente. $P / E=1.82$. Exina de $2.5 \mu$ de grosor, con la sexina de $1.5 \mu$ de espesor y la nexina de $\pm 1 \mu$; superficialmente al MEB y ML se aprecia reticulada, con lúmenes de \pm $2 \mu$. Colpos de 33 a $38 \mu$ de largo por $1.5 \mu$ de ancho. Colpos transversos de 4.5 a 6 X 3 a $4 \mu$. Indice del área polar 0.85 , muy grande. 
Luehea candida (DC.) Mart.

Estación de Biología Chamela, Jalisco.

E. Lott 496 (MEXU).

Lám. VIII. Figs. 1 a 4.

Polen tricolporado, tectado, subprolato a prolato de 66 (69) $72 \times 49$ (51.5) $55 \mu$. Vista polar poco frecuente. $P / E=1.33$. Exina de 3 a $3.3 \mu$ de grosor, con la sexina de 2.5 $\mu$ de espesor y la nexina menor de $1 \mu$; superficialmente al MEB y ML se aprecia reticulada, con lúmenes de $\pm 1 \mu$. Colpos de 55 a $59 \mu$ de largo por 1.5 a $2 \mu$ de ancho. Poros de 2.5 a $3.5 \mu$ de diámetro.

Triumfetta acracantha Hochr.

Estación de Biología Chamela, Jalisco.

S. H. Bullock 1462 (MEXU).

Lám. VIII. Figs. 5 a 8.

Polen tricolporado, tectado, prolato de 37 (42.5) $46.5 \times 22$ (24) $27 \mu$. Vista polar poco frecuente. $P / E=1.7$. Exina de $2.5 \mu$ de grosor, con la sexina de $1.5 \mu$ de espesor y la nexina de $\pm 1 \mu$; superficialmente al MEB reticulada con puntuaciones dentro de los lúmenes; lúmenes de $\pm 2 \mu$ de diámetro, superficialmente al $\mathrm{ML}$ se aprecia reticulada. Colpos de 29 a $34 \mu$ de largo por 1.5 a $2 \mu$ de ancho. Colpos transversos de 4 a $5 \times 2$ a $3 \mu$. Indice del área polar 0.71 , grande.

Clave para diferenciar las especies de la familia Tiliaceae

1. Granos de polen reticulados, con lúmenes de $\pm 2 \mu$ de diámetro Heliocarpus pallidus Lám. VII. Figs. 9 a 12 Triumfetta acracantha Lám. VIII. Figs. 5 a 8

1. Granos de polen reticulados, con lúmenes de $\pm 1 \mu$ de diámetro o menores Luehea candida Lám. VIII. Figs. 1 a 4

Comentarios

Erdtman (1966) distingue 3 grupos en las Tiliaceae, el tipo Tilia con polen tricolporado, colpos cortos, peroblato a suboblato con ornamentación puntitegilada o escabrosa; el tipo Grewia con granos tricolporados, pero a diferencia del conjunto anterior los colpos son largos y la sexina reticulada; y el último grupo palinológico llamado otros tipos, donde se incluyen géneros con granos de polen muy heterogéneos, por ejemplo porado y equinado, parecido al polen de malváceas o bien dispuesto en tétradas tetraédricas.

Por las características del polen de las especies de Tiliaceae de la Estación de Biología Chamela, Jalisco, los tres géneros corresponderían al tipo Grewia. 
Familia Violaceae

Taxonomía: Lott (1985).

Palinología: Erdtman (1943), Faegri \& Iversen (1964), Heusser (1971), Kapp (1969), Plá Dalmau (1961).

Hybanthus attenuatus

(Humb. \& Bonpl.) G. K.

Schulze
Estación de Biología Chamela, Jalisco. A. Pérez 1903 (MEXU). Lám. IX. Fig. 1 a 5.

Polen tricolporado, tectado, subprolato de 24.5 (26) $30 \times 19.5$ (20) $21 \mu$. Vista polar circular a subtriangular de 24.5 (27.5) $31.5 \times 21$ (23) $25 \mu$. P/E= 1.23. Exina de $2 \mu$ de grosor, con la sexina de $1.5 \mu$ de espesor y la nexina menor de $1 \mu$; superficialmente al MEB se aprecia granular y al ML psilada a levemente granular. Colpos de 18 a $21 \mu$ de largo por $1.5 \mu$ de ancho con membranas escabrosas. Poros de 1.5 a $2 \mu$ de diámetro. Indice del área polar 0.64, grande.

Hybanthus mexicanus Ging.

Estación de Biología Chamela, Jalisco. S. H. Bullock 1186 (MEXU).

Lám. IX. Figs. 6 a 9.

Polen tricolporado, tectado, esferoidal de 17 (19) $21 \mu$ de diámetro. Vista polar circular de 16 (19) $21 \mu$ de diámetro. $P / E=1.08$. Exina de $2.5 \mu$ de grosor con la sexina de $1.5 \mu$ de espesor y la nexina de $\pm 1 \mu$; superficialmente al MEB se aprecia granular con puntuaciones y al ML psilada. Colpos de 13 a $15 \mu$ de largo por $1.5 \mu$ de ancho. Poros de 1.5 a $2 \mu$ de diámetro. Indice del área polar 0.86, muy grande.

Hybanthus serrulatus Standl.

Estación de Biología Chamela, Jalisco.

Lám. IX, Figs. 10 a 13. J. A. Solís-Magallanes 4304 (MEXU).

Polen tricolporado, tectado, subprolato de 24.5 (26) $28 \times 20$ (22) $23.5 \mu$. Vista polar semiangular de 28.5 (30) $31.5 \times 27$ (28) $29.5 \mu$. P/E=1.18. Exina de $2 \mu$ de grosor con la sexina de $1.5 \mu$ de espesor y la nexina menor de $1 \mu$; superficialmente al MEB se aprecia granular con puntuaciones y al ML psilada. Colpos de 18 a $21 \mu$ por 1.5 a $2 \mu$ de ancho. Poros de 2 a $3 \mu$ de diámetro. Indice del área polar 0.69 , grande.

\section{Comentarios}

Los diferentes autores que han descrito el polen de Violaceae, lo consideran muy homogéneo, tricolporado y con la ornamentación reticulada, psilada o escabrosa, características que se presentan en la mayor parte de las plantas de esta familia, de tal manera, que resulta difícil la separación tanto genérica como específica. En el caso particular de los tres representantes de Hybanthus de la Estación de Biología Chamela, Jalisco, no fue posible su diferenciación por medios palinológicos. 


\section{AGRADECIMIENTOS}

Damos gracias al Dr. S. H. Bullock de la Estación de Biología de Chamela, Jal., el envío de las muestras florales para su procesamiento. Así también, deseamos expresar nuestro agradecimiento al Colegio de Posgraduados de Chapingo, por permitir el uso del microscopio electrónico de barrido y al M. en C. Jorge Valdez por toda la ayuda y asesoramiento que nos ha brindado en el trabajo.

\section{LITERATURA CITADA}

Association des Palinologues de Langue Française. 1974. Pollen et spores d'Afrique tropicale. Centre d'Etudes de Géographie Tropicale. Domaine Universitaire de Bordeaux. Talance. 283 pp.

Barth, O. M. \& S. A. F. Silva. 1965. Catálogo sistemático dos polen das plantas arboreas do Brasil Meridional VII. Thymelaeaceae, Lythraceae, Lecythidaceae, Rhizophoraceae e Combretaceae. Mem. Inst. Oswaldo Cruz 63: 255-278.

Erdtman, G. 1943. An introduction to pollen analysis. The Ronald Press Co. Nueva York. 239 pp.

Erdtman, G. 1966. Pollen morphology and plant taxonomy. Angiosperms. Hafner Publishing Co. Nueva York. 553 pp.

Faegri, K. \& J. Iversen. 1964. Textbook of pollen analysis. Hafner Publishing Co. Nueva York. 273 pp.

Graham, A. 1980. Morfología del polen de Eugenia-Myrcia (Myrtaceae) y Combretum-Terminalia (Combretaceae) en relación a su alcance estratigráfico en el Terciario del Caribe. Biotica 5(1): 5-13.

Heusser, C. J. 1971. Pollen and spores of Chile. The University of Arizona Press. Tucson, Arizona. $167 \mathrm{pp}$.

Huang, T. C. 1972. Pollen flora of Taiwan. National Taiwan University Botany Department Press. Taipei. $276 \mathrm{pp}$.

Jain, R. K. \& S. Nanda. 1966. Pollen morphology of some deserts plants of Pilania, Rajasthan. Palynological Bull. Lucknow 2-3: 56-69.

Kapp, R. O. 1969. How to know pollen and spores. W.C. Brown Co. Dubuque, lowa. 249 pp.

Kubitzki, K. 1965. Palynologia Madagassica et Mascarenica: Fam. 147-254. Lythraceae-Oenotheraceae. Pollen et Spores 7(3): 491-507.

Lott, E. J. 1985. Listados florísticos de México III. La Estación de Biología Chamela, Jalisco. Instituto de Biología, Universidad Nacional Autónoma de México. México, D.F. 47 pp.

Ludlow-Wiechers, B. \& L. Roldán-Ramos. 1984. Catálogo palinológico para la flora de Veracruz 20. Familia Martyniaceae. Biotica 9(4): 407-414.

Maley, J. 1970. Contributions à l'étude de Bassin Tchadien. Atlas de pollen du Tchad. Bull. Jard. Bot. Nat. Belg. 40: 29-48.

Markgraf, V. \& H. D’Antoni. 1978. Pollen flora of Argentina. The University of Arizona Press. Tucson, Arizona. 208 pp.

Melhen, T. S. \& J. E. De Paula. 1966. Pollen grains of plants of the "Cerrado" XIII. Celastraceae, Combretaceae and Rosaceae. An. Academ. Brasil C. I. 38(1): 205-216.

Moncada, M. 1987. Morfología del polen de Amaranthaceae. Acta Botánica Cubana 44: 1-8.

Nair, P. K. \& M. Sharma. 1962. Pollen morphology with reference to the geographical distribution of the genus Argemone mexicana. Lloydia 25(2): 3-129.

Nowicke, J. W. \& J. Skvarla. 1979. Pollen morphology: The potential influence in higher older systematics. Ann. Missouri Bot. Gard. 66: 633-700.

Ownbey, G. B. 1958. Monograph of the genus Argemone for North America and the West Indies. Mem. Torr. Bot. Club 21: 1-59. 
Palacios, Ch., R. 1966. Morfología de los granos de polen de árboles del Estado de Morelos. An. Esc. Nac. Cienc. Biól. Méx. 16: 41-169.

Palacios-Chávez, R., D. L. Quiroz-García, M. L. Arreguín-Sánchez \& D. Ramos-Zamora. 1986. Flora polínica del bosque tropical caducifolio de Chamela, Jalisco. México. Presentación. Phytologia 61(3): 147-149.

Plá Dalmau, J. M. 1961. Polen. Talleres Gráficos D.C.P. Gerona. 526 pp.

Punt, W. \& P. W. Leenhouts. 1967. Pollen morphology and taxonomy in the Loganiaceae. Grana 7(23): 469-516.

Riollet, G. \& R. Bonnefille. 1976. Pollen des Amaranthacées du Bassin du lac Rodolphe (Afrique Orientale). Déterminations générique et spécifique. Pollen et Spores 18(1): 67-92.

Schinz, H. 1934. Amaranthaceae. In: Engler \& Prantl, Die natürlichen Pflanzenfamilien, ed. 2, 16c. pp. 7-85.

Tsukada, M. 1967. Chenopodiaceae and Amaranthaceae pollen: electron microscopic determination. Science 157: 81.

Valio, I. F. M. \& M. L. S. Labouriau. 1964. Pollen grains of plants of the "Cerrado" VII. Liliaceae, subfamily Smilacoideae and Loganiaceae. Rev. Bras. Biol. 24(2): 119-126. 


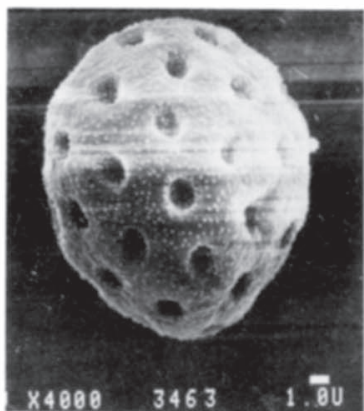

1



2



3

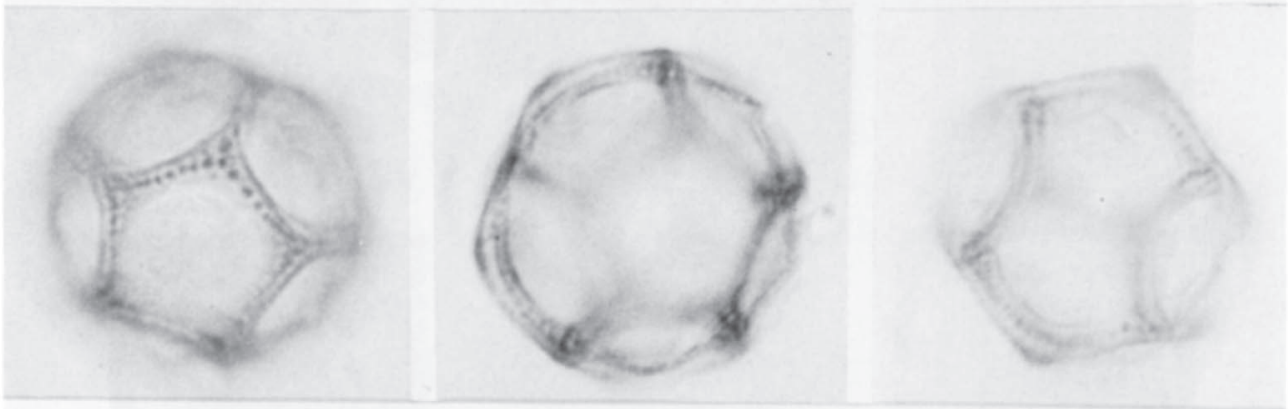

4

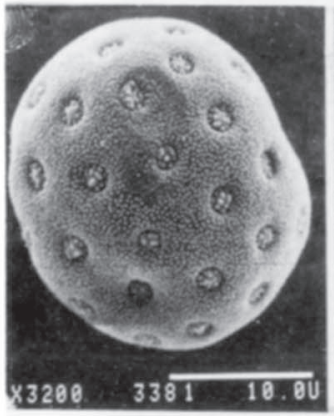

7

8

5

6

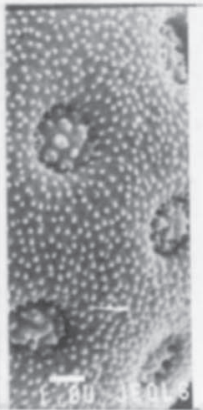

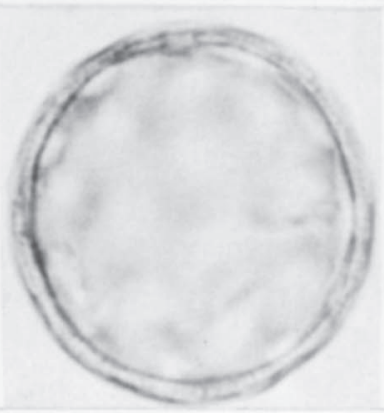

9

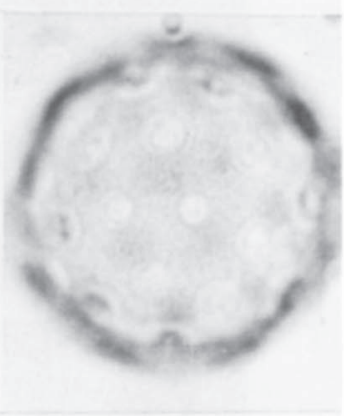

10

Lámina I. Achyrantes aspera. 1. Vista general del grano de polen al MEB; 2. Sección óptica; 3. Vista superficial que muestra ornamentación y poros. Alternanthera cf. pycnantha. 4. Vista superficial, se observan las microespinas sobre los muros; 5 y 6 . Sección óptica. Amaranthus retroflexus. 7. Vista general del grano de polen al MEB; 8. Acercamiento superficial al MEB; 9. Sección óptica; 10. Vista superficial. 

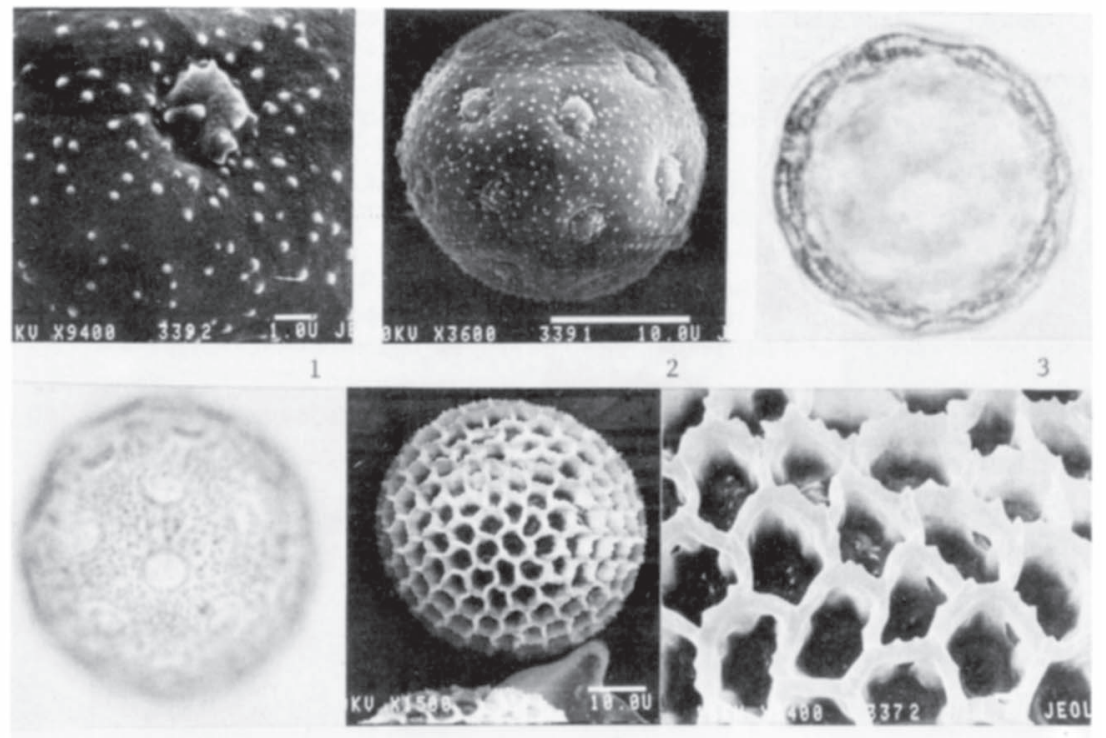

4

5

6



7

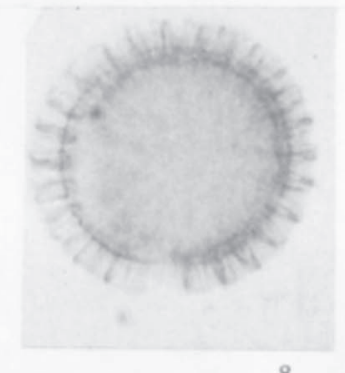

8
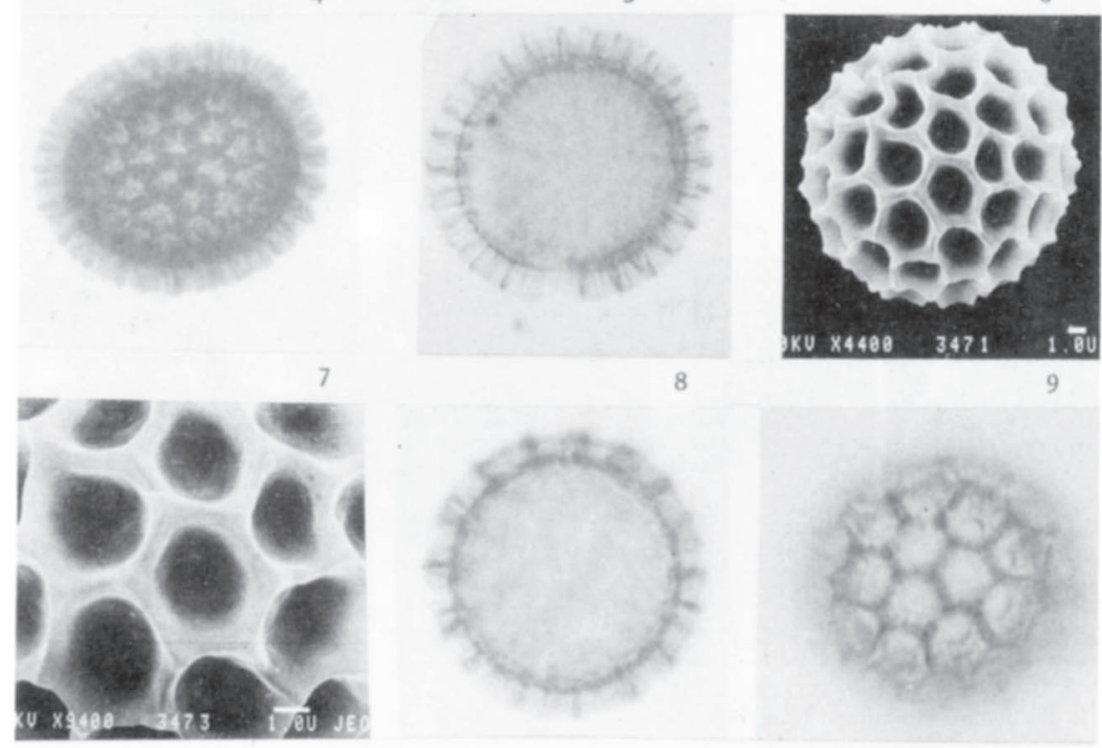

9

10

11

12

Lámina II. Chamissoa altissima. 1. Acercamiento superficial al MEB, muestra opérculo y microespinas; 2. Vista total del grano de polen al MEB; 3. Sección óptica; 4. Vista superficial. Froelichia interrupta. 5. Vista general del grano de polen al MEB; 6. Acercamiento superficial al MEB; 7. Vista superficial; 8. Sección óptica. Gomphrena decumbens. 9. Vista total del grano de polen al MEB; 10. Acercamiento superficial al MEB; 11. Sección óptica; 12. Vista superficial. 


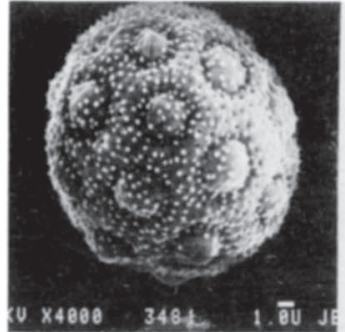

1

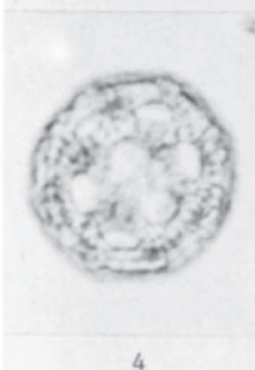

4

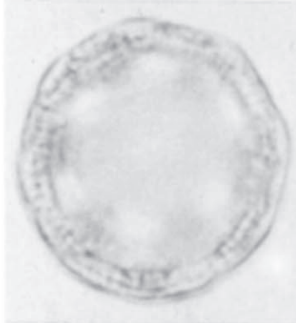

7



10

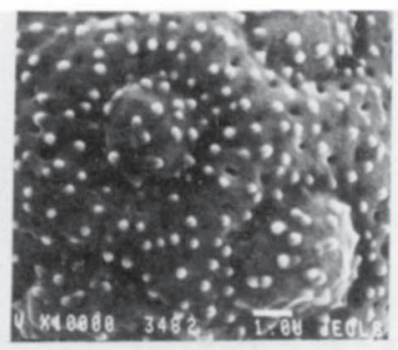

2

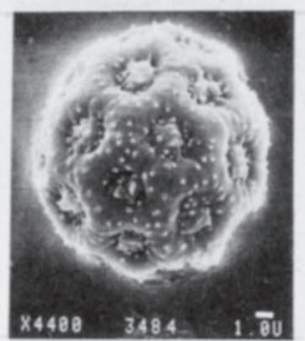

5

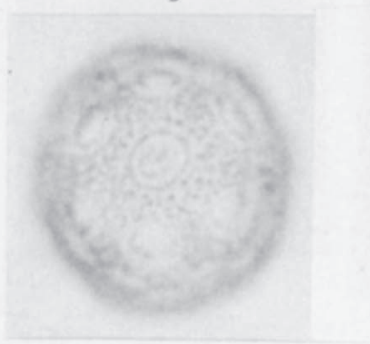

8

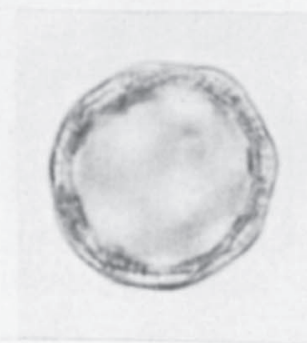

11

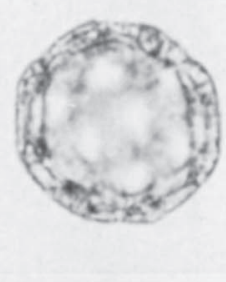

3

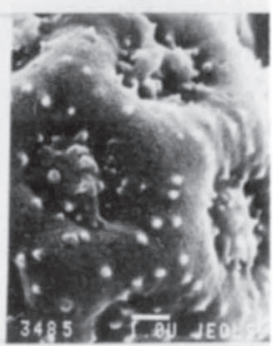

6

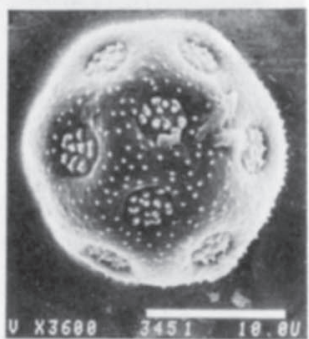

9

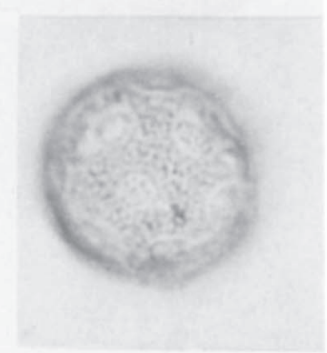

12

Lámina III. Iresine calea. 1. Vista total del grano de polen al MEB; 2. Acercamiento superficial al MEB; 3. Sección óptica; 4. Vista superficial. Iresine interrupta. 5. Vista general del grano de polen al MEB; 6. Acercaminento superficial al MEB; 7. Sección óptica; 8. Vista superficial. Lagrezia monosperma. 9. Vista total del grano de polen al MEB; 10. Acercamiento superficial al MEB; 11. Sección óptica; 12. Vista superficial. 




Lámina IV. Combretum fruticosum. 1. Vista ecuatorial al MEB; 2. Detalle de la ornamentación al MEB; 3. Vista ecuatorial mostrando los colpos y ornamentación; 4. Grosor de la exina; 5. Vista polar al MEB; 6. Vista polar mostrando los colpos y pseudocolpos; 7. Vista polar. Combretum mexicanum. 8. Vista ecuatorial al MEB, mostrando colpos y ornamentación; 9. Vista ecuatorial; 10. Grosor de la exina; 11. Vista polar al MEB; 12. Vista polar mostrando colpos y pseudocolpos; 13. Detalle de la ornamentación al MEB; 14. Vista polar. 
Quiroz-García et al.: Flora Polínica de Chamela, Jalisco

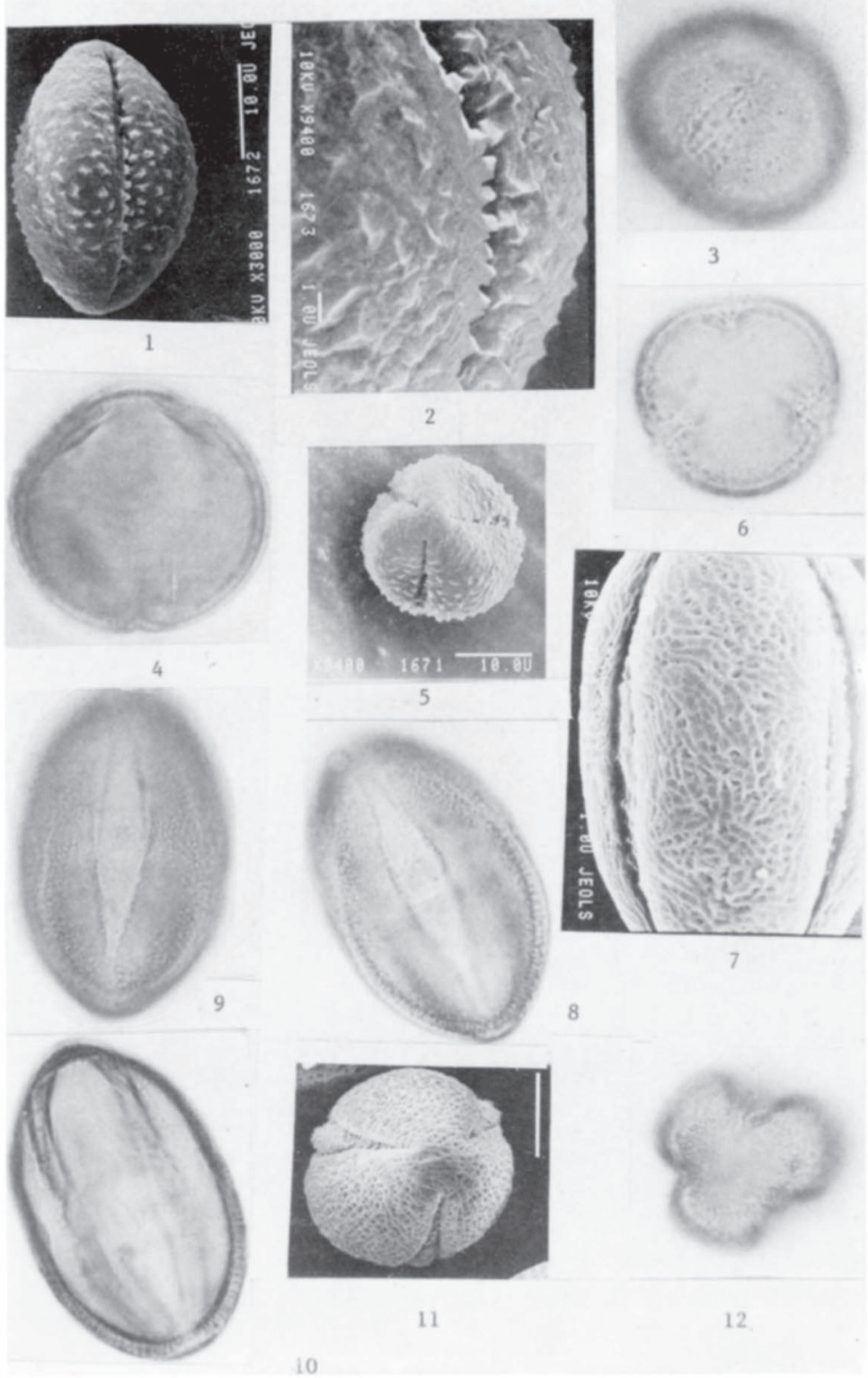

Lámina V. Gronovia scandens. 1. Vista ecuatorial al MEB; 2. Detalle de la ornamentación y colpo al MEB; 3. Vista ecuatorial mostrando la ornamentación; 4. Vista ecuatorial mostrando grosor de la exina; 5. Vista polar al MEB; 6. Vista polar. Mentzelia aspera. 7. Detalle de la vista ecuatorial al MEB; 8. Vista ecuatorial mostrando los colpos y grosor de la exina; 9. Vista ecuatorial mostrando detalle de la ornamentación; 10. Vista ecuatorial; 11. Vista polar al MEB; 12. Vista polar. 


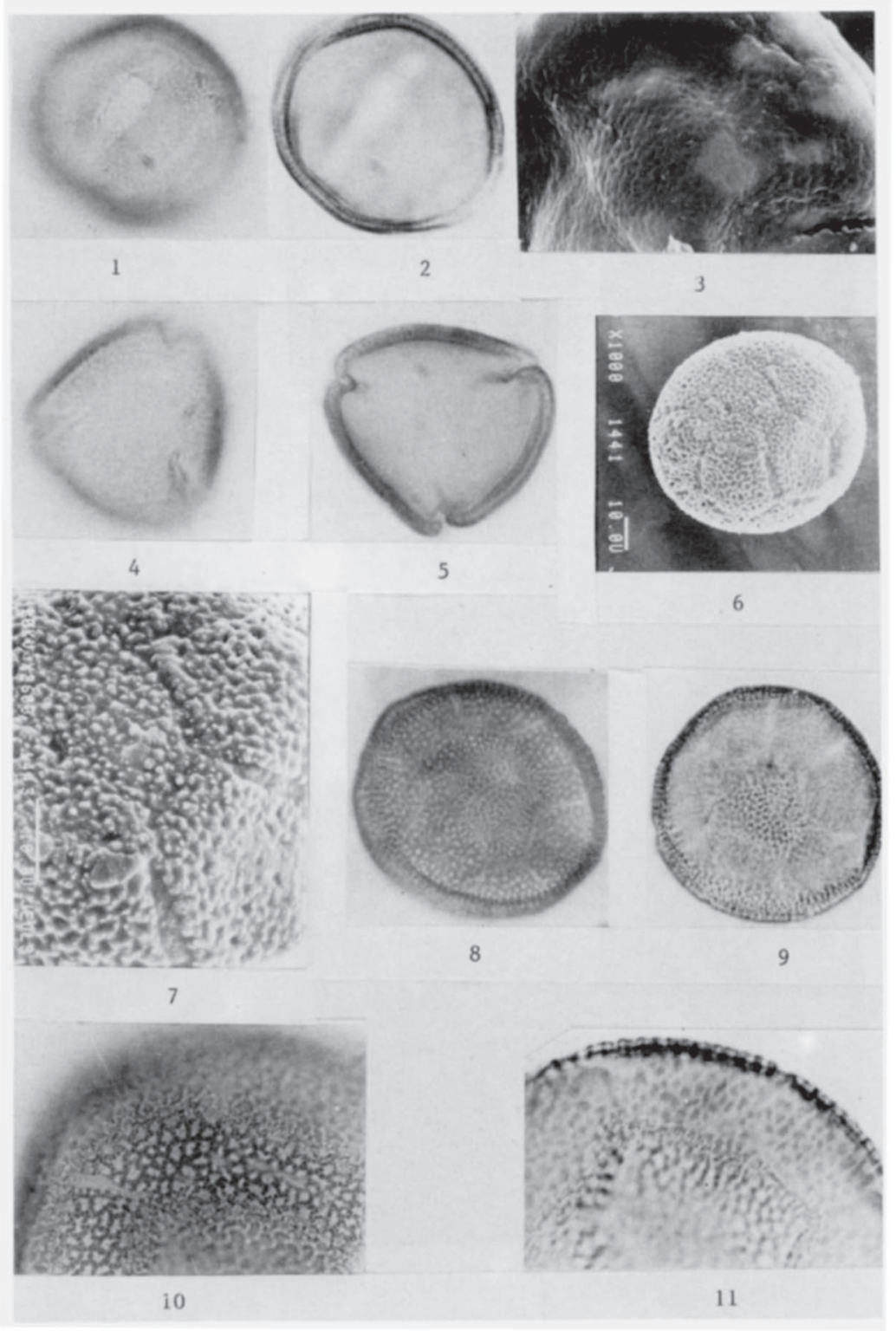

Lámina VI. Strychnos cf. brachistantha. 1. Vista ecuatorial mostrando un colpo; 2. Vista ecuatorial mostrando grosor de la exina; 3. Detalle de la ornamentación al MEB; 4. Vista polar mostrando la ornamentación; 5. Vista polar, grosor de la exina. Martynia annua. 6. Polen al MEB mostrando varios colpos; 7. Detalle de la ornamentación al MEB; 8. Vista superficial mostrando los colpos; 9. Grosor de la exina; 10. Detalle de un colpo y ornamentación; 11. Detalle del grosor de la exina. 


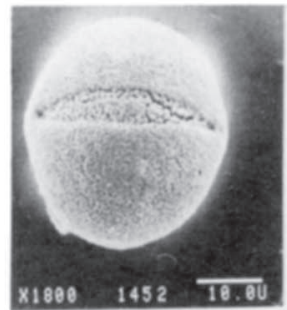

1

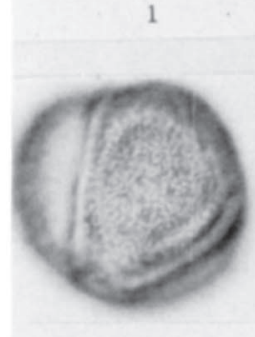

4

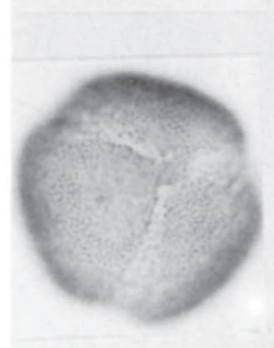

7

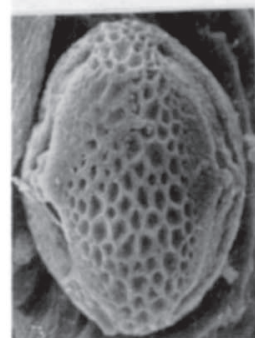

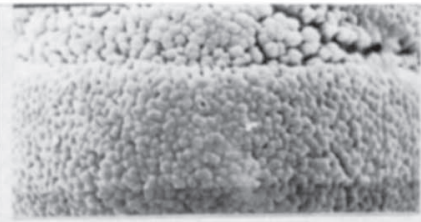

2

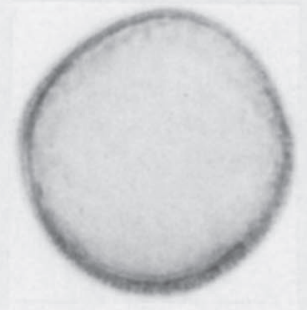

5

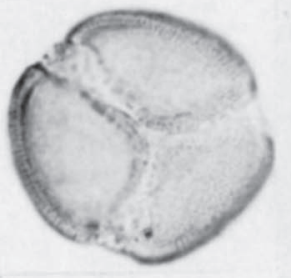

8
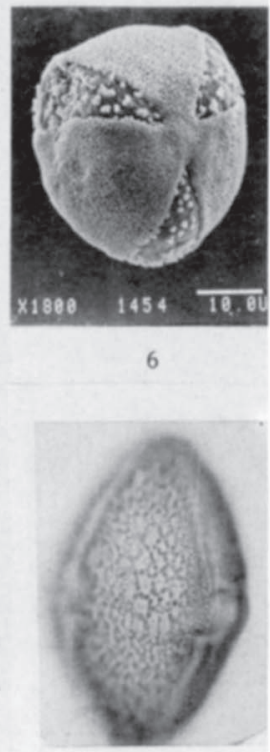

9



11

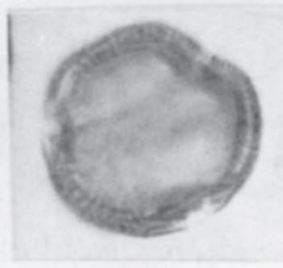

12

Lámina VII. Argemone ochroleuca subsp. ochroleuca. 1. Vista ecuatorial al MEB; 2. Detalle de la ornamentación al MEB; 3. Vista ecuatorial mostrando detalle de la ornamentación; 4 . Detalle de los colpos y ornamentación; 5. Grosor de la exina; 6 . Vista polar al MEB; 7. Vista polar mostrando colpos y ornamentación; 8. Vista polar mostrando grosor de la exina. Heliocarpus pallidus. 9. Vista ecuatorial mostrando colpos y ornamentación; 10. Vista ecuatorial al MEB; 11. Detalle de la ornamentación al MEB; 12. Vista polar. 




4

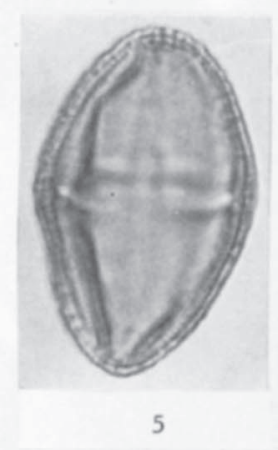

5

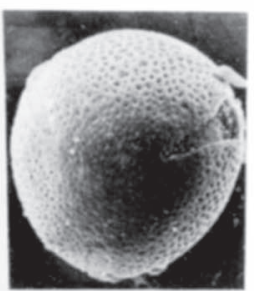

3



6
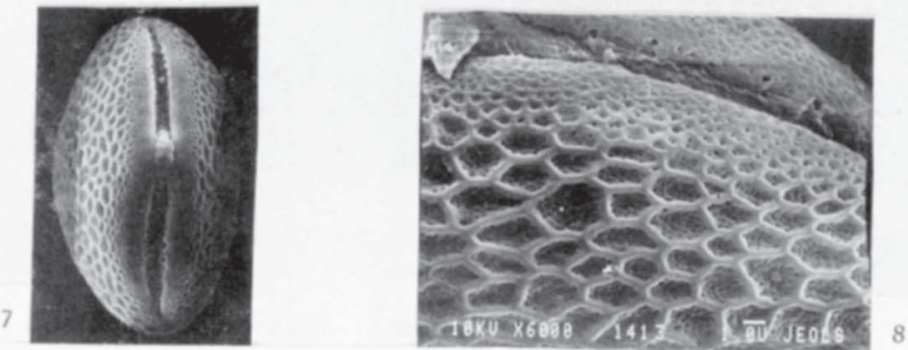

Lámina VIII. Luehea candida. 1. Vista ecuatorial superficial mostrando los colpos; 2. Vista ecuatorial al MEB; 3. Vista polar al MEB; 4. Detalle de la ornamentación al MEB; Triumfetta acracantha. 5. Vista ecuatorial mostrando el grosor de la exina; 6 . Vista ecuatorial, ornamentación; 7. Vista ecuatorial al MEB; 8. Detalle de la ornamentación al MEB. 
Quiroz-García et al.: Flora Polínica de Chamela, Jalisco

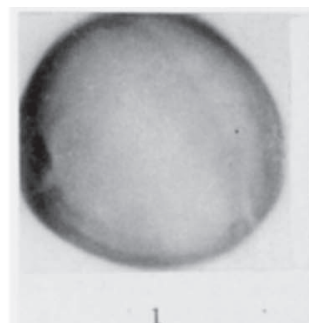

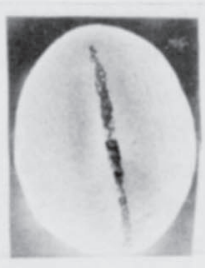

2

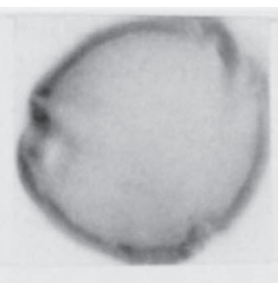

3



4
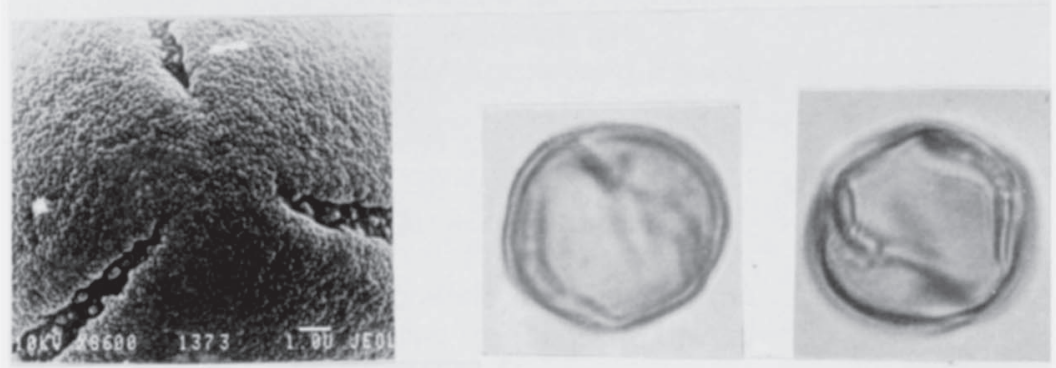

5

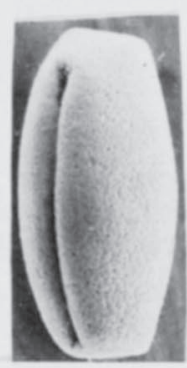

6

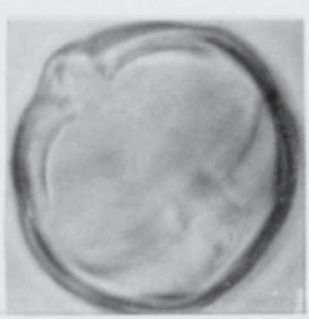

10

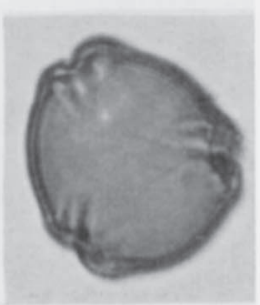

9

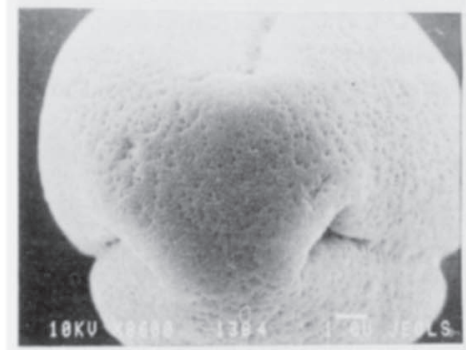

12

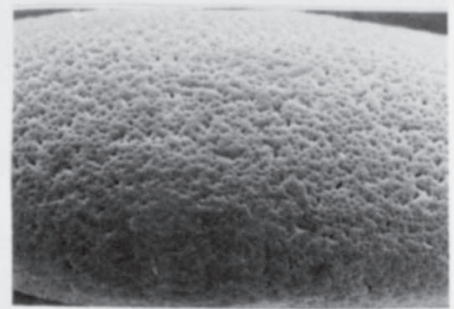

13

Lámina IX. Hybanthus attenuatus. 1. Vista ecuatorial; 2. Vista ecuatorial al MEB; 3. Vista polar; 4. Vista polar al MEB; 5. Detalle de la ornamentación al MEB. Hybanthus mexicanus. 6. Vista ecuatorial; 7. Detalle de un colpo y un poro; 8. Vista polar; 9. Vista ecuatorial al MEB. Hybanthus serrulatus. 10. Vista ecuatorial mostrando colpos y poros; 11. Vista polar; 12. Vista polar al MEB; 13. Detalle de la ornamentación al MEB. 